九州大学学術情報リポジトリ

Kyushu University Institutional Repository

\title{
Light Curve Approximation Using an Attitude Model of Solar Sail Spacecraft
}

Yoshimura, Yasuhiro

Department of Aeronautics and Astronautics, Kyushu University : Assistant Professor

Matsushita, Yuri

Department of Aeronautics and Astronautics, Kyushu University : Graduate Student

Arakawa, Ryohe i

Department of Aeronautics and Astronautics, Kyushu University : Graduate Student

Hanada, Toshiya

Department of Aeronautics and Astronautics, Kyushu University : Professor

http://hdl. hand le. net/2324/4481550

出版情報: Journal of Guidance, Control, and Dynamics. 43 (10), pp.1960-1966，2020-10. American Institute of Aeronautics and Astronautics

バージョン :

権利関係 : 


\title{
Light Curve Approximation Using an Attitude Model of Solar Sail Spacecraft
}

\author{
Yasuhiro Yoshimura*, Yuri Matsushita ${ }^{\dagger}$, Ryohei Arakawa $^{\ddagger}$, and Toshiya Hanada ${ }^{\S}$. \\ Kyushu University, Fukuoka 819-0395, Japan
}

\begin{abstract}
I. Introduction
Space Situational Awareness (SSA) is an emergent issue to properly understand space environment and its future projections [1, 2]. Ground-based optical measurement is a cost-effective approach to observe space objects from the ground. Photometric data obtained by the observations, called light curves, play a significant role in estimating object states. Light curves are the magnitude changes of the reflected light from an object surface to an observer, and provide the information about the geometry among the Sun, the object, and the observer, i.e., their relative positions. Furthermore, light curves include various types of information such as attitude, shape, and surface properties of space objects, which are extracted for the state estimation. The various information in light curves, however, indicate that the number of state variables for the estimation tends to be large. The light curve data, on the other hand, is scalar data as the time history of brightness of the space object, and the state estimation with light curve data is still a challenging problem due to low observability compared to the large number of state variables to be estimated.

Light curves are formulated using Bidirectional Reflectance Distribution Functions (BRDFs), which describe the ratio of light intensity between irradiance and radiance. In other words, BRDFs parameterize the optical property of an object surface. There are three BRDFs often used for light curve research. The simplest BRDF is parameterized with Lambertian diffuse and perfect mirror-like specular. This model has been widely used in research on light curves as well as Solar Radiation Pressure (SRP) modelling [3]. The current study also uses this model due to the compatibility to the formulation of solar radiation pressure. Cook-Torrance model [4, 5] has Lambertian diffuse and the directional distributions of specular reflection. Ashikhmin-Shirley model [6] includes non-Lambertian diffuse and anisotropic reflectance as used in [7]. Although there is no consensus of BRDF to be used. since each component of space objects has different values of the optical parameters, estimating all parameters is not practically reasonable.

SRP and its torque are one of main disturbances that contribute to spacecraft translational and rotational motion, which are also formulated with BRDFs. In [7], differences due to BRDFs used are discussed and refined with a correction parameter. By expressing an albedo term common in light curves and SRP, the translational motion of a space object is estimated in [8]. On the other hand, the dynamics of solar sail spacecraft, in which SRP is dominant
\end{abstract}

\footnotetext{
*Assistant Professor, Department of Aeronautics and Astronautics, 744 Motooka, Nishi-ku, Fukuoka, Japan.

${ }^{\dagger}$ Graduate Student,Department of Aeronautics and Astronautics, 744 Motooka, Nishi-ku, Fukuoka, Japan.

${ }^{\ddagger}$ Graduate Student, Department of Aeronautics and Astronautics, 744 Motooka, Nishi-ku, Fukuoka, Japan.

${ }^{\S}$ Professor, Department of Aeronautics and Astronautics, 744 Motooka, Nishi-ku, Fukuoka, Japan. Member AIAA
} 
external force, has been intensively studied and its generalized formulation has been compared with on-orbit data [9, 10]. The generalized formulation of solar sail spacecraft in [10, 11] can express the shape and optical parameters of the spacecraft with three parameters without loss of physical meanings. Since the formulations of light curves and the dynamics of solar sail spacecraft are related via BRDFs, the rotational motion of a space object also should be expressed with common terms in light curves and SRP torques.

In this context, this study aims to explicitly combine the photometric formulation of a space object with the dynamics of solar sail spacecraft. The formulation is derived using spin averaging and enables not only approximating light curves with a fewer number of parameters, but also expressing the surface properties and the light curves explicitly in the attitude dynamics of the space object. In other words, the surface properties, the light curves, and the attitude dynamics are physically related to one another in the formulation. Although the light curve formulation is not exactly the same as the parameter in the dynamics of the solar sail spacecraft, multiplying a correction factor enables compensating the difference.

\section{Formulations}

Both light curves and SRP are caused by sunlight and its reflection, and they are formulated with BRDFs. This section describes the formulations of a BRDF model, light curves, and SRP. These are geometrically described on a small facet that is obtained by splitting an arbitrary shape object into many small facets. The sum of them provides the high-fidelity calculation of light curves and SRP.

\section{A. Bidirectional Reflectance Distribution Function (BRDF)}

A BRDF $f_{r}$ describes the light intensity ratio between irradiance $L_{i}$ and reflected radiance $L_{r}$ as:

$$
f_{r}=\frac{L_{r}}{L_{i}}
$$

The geometry of the reflected light on a small facet is illustrated in Fig. 1, where $\boldsymbol{n}$ is the normal vector of the facet. The vectors $\boldsymbol{s}=\left[s_{x}, s_{y}, s_{z}\right]^{T}$ and $\boldsymbol{v}=\left[v_{x}, v_{y}, v_{z}\right]^{T}$ are the sun directional vector and the reference vector, which are defined with sets of azimuth and elevation angles $\left(\phi_{i}, \theta_{i}\right)$ and $\left(\phi_{r}, \theta_{r}\right)$, respectively. The reflected light is parameterized with diffuse and specular reflections as

$$
f_{r}=d c_{d}+s c_{s}
$$

where $d$ and $s$ are the fraction of the diffusion and specularity, respectively, and they satisfy $d+s=1$. It should be noted that, in general, the coefficients $c_{d}$ and $c_{s}$ are the function of $\theta_{r}$ and $\phi_{r}$. Thus, the total coefficients of diffuse and 




Fig. 1 Light reflection on a facet

specular reflections are obtained by integrating over the hemisphere around the normal vector $\boldsymbol{n}$ as

$$
\begin{gathered}
c_{\text {diff }}=\iint d c_{d} \cos \theta_{r} \sin \theta_{r} \mathrm{~d} \theta_{r} \mathrm{~d} \phi_{r} \\
c_{\text {spec }}=\iint s c_{s} \cos \theta_{r} \sin \theta_{r} \mathrm{~d} \theta_{r} \mathrm{~d} \phi_{r}
\end{gathered}
$$

These coefficients satisfy the following inequality for energy conservation.

$$
c_{\mathrm{diff}}+c_{\mathrm{spec}} \leq 1
$$

If the sum of them is equal to one, the facet has neither absorption nor transparent. If the sum is less than one, the residual energy will be absorbed and/or passed through. Furthermore, a part of the absorbed energy is thermally emitted, which also accelerates the facet.

A typical BRDF is assumed to be Lambertian diffuse and perfect mirror-like specular, and such BRDF is described as

$$
f_{r}=d \frac{\rho}{\pi}+s\left[2 F_{0} \delta\left(\sin ^{2} \theta_{i}-\sin ^{2} \theta_{r}\right) \delta\left(\phi_{i}-\phi_{r} \pm \pi\right)\right]
$$

where $\rho$ is the diffuse reflectance, $F_{0}$ is the Fresnel reflectance, and $\delta(\cdot)$ is the Dirac delta function. For this simple BRDF, the integrations in Eqs. (3) and (4) can be analytically calculated as

$$
\begin{gathered}
c_{\text {diff }}=d \rho \\
c_{\text {spec }}=s F_{0}
\end{gathered}
$$


These are equivalently written as follows.

$$
\begin{gathered}
\frac{c_{\text {diff }}}{\pi}=d c_{d} \\
2 c_{\text {spec }} F_{0} \delta\left(\sin ^{2} \theta_{i}-\sin ^{2} \theta_{r}\right) \delta\left(\phi_{i}-\phi_{r} \pm \pi\right)=s c_{s}
\end{gathered}
$$

The relations in Eqs. 9) and (10) are used to relate the light curve formulation to the dynamics of solar sail spacecraft.

Although this paper deals with the simple BRDF in Eq. (6) for light curves and SRP, there are other BRDFs that can express more detailed reflections. Cook-Torrance model [4] expresses the Lambertian diffuse and specular reflections due to microfacets. The BRDF is described as

$$
\begin{aligned}
c_{d} & =\frac{\rho}{\pi} \\
c_{s} & =\frac{D G F}{4\left(\boldsymbol{n}^{T} \boldsymbol{s}\right)\left(\boldsymbol{n}^{T} \boldsymbol{v}\right)}
\end{aligned}
$$

where $D$ is the facet slope distribution function, $G$ is the geometrical attenuation factor, and $F$ is the Fresnel reflection term. These terms are written as

$$
\begin{aligned}
D & =\frac{1}{\pi m^{2} \cos ^{4} \alpha} e^{-\left(\frac{\tan \alpha}{m}\right)^{2}} \\
G & =\min \left\{1, \frac{2\left(\boldsymbol{n}^{T} \boldsymbol{h}\right)\left(\boldsymbol{n}^{T} \boldsymbol{v}\right)}{\boldsymbol{v}^{T} \boldsymbol{h}}, \frac{2\left(\boldsymbol{n}^{T} \boldsymbol{h}\right)\left(\boldsymbol{n}^{T} \boldsymbol{s}\right)}{\boldsymbol{v}^{T} \boldsymbol{h}}\right\} \\
F & =\frac{\left(g-\boldsymbol{v}^{T} \boldsymbol{h}\right)^{2}}{2\left(g+\boldsymbol{v}^{T} \boldsymbol{h}\right)^{2}}\left\{1+\frac{\left[\boldsymbol{v}^{T} \boldsymbol{h}(g+\boldsymbol{v} \boldsymbol{h})-1\right]^{2}}{\left[\boldsymbol{v}^{T} \boldsymbol{h}(g-\boldsymbol{v} \boldsymbol{h})+1\right]^{2}}\right\}
\end{aligned}
$$

where

$$
\begin{aligned}
\boldsymbol{h} & =\frac{\boldsymbol{s}+\boldsymbol{v}}{\|\boldsymbol{s}+\boldsymbol{v}\|} \\
g^{2} & =n_{\mathrm{est}}^{2}+\left(\boldsymbol{v}^{T} \boldsymbol{h}\right)^{2}-1 \\
n_{\mathrm{est}} & =\frac{1+\sqrt{F_{0}}}{1-\sqrt{F_{0}}}
\end{aligned}
$$

The parameter $m$ means the spread of specular reflections. The bisector vector $\boldsymbol{h}$ is defined with $\boldsymbol{s}$ and $\boldsymbol{v}$ and whose azimuth and elevation angles are $\beta$ and $\alpha$, respectively. That is, $\boldsymbol{h}=[\sin \alpha \cos \beta, \sin \alpha \sin \beta, \cos \alpha]^{T}$. 
Ashikhmin-Shirley model [6] is an isotropic BRDF, and it is written as

$$
\begin{aligned}
c_{d} & =\frac{28 \rho}{23 \pi}\left(1-s F_{0}\right)\left[1-\left(1-\frac{\boldsymbol{n}^{T} \boldsymbol{s}}{2}\right)^{5}\right]\left[1-\left(1-\frac{\boldsymbol{n}^{T} \boldsymbol{v}}{2}\right)^{5}\right] \\
c_{s} & =\frac{\sqrt{\left(n_{u}+1\right)\left(n_{v}+1\right)}}{8 \pi} \frac{F}{\boldsymbol{v}^{T} \boldsymbol{h} \max \left\{\boldsymbol{n}^{T} \boldsymbol{s}, \boldsymbol{n}^{T} \boldsymbol{v}\right\}}(\cos \alpha)^{n_{u} \cos ^{2} \beta+n_{v} \sin ^{2} \beta}
\end{aligned}
$$

where $n_{u}$ and $n_{v}$ are exponential factors that define the shape of specular lobe. The Fresnel reflectance $F$ is written as follows.

$$
F=F_{0}+\left(\frac{1}{s}-F_{0}\right)\left(1-\boldsymbol{v}^{T} \boldsymbol{h}\right)^{5}
$$

Cook-Torrance model and Ashikhmin-Shirley model have more complicated specular reflections as shown in Eqs. (12) and 20, than the simple BRDF. Thus analytical integrations in Eqs. (3) and (4) cannot be obtained for Cook-Torrance model and Ashikhmin-Shirley model, which limits the applicability of the proposed method in this paper to the simple BRDF.

\section{B. Light curves}

Light curves are successive photometric data obtained by ground-based observations and their intensity depends on incoming light and reflected light. The relative magnitude of the brightness of a facet is described as follows.

$$
m_{\mathrm{app}}=m_{\mathrm{sun}}-2.5 \log _{10} \frac{f_{\mathrm{obs}}}{r_{\mathrm{obs}}^{2}}
$$

where $m_{\text {sun }}=-26.7$ in this paper, $r_{\text {pos }}$ is the distance between the object and the observer, and

$$
f_{\text {obs }}=c_{\text {total }} f_{i} A\left(\boldsymbol{n}^{T} \boldsymbol{v}\right)
$$

In Eq. 23, $c_{\text {total }}=d c_{d}+s c_{s}, A$ is the area of the facet, and the function $f_{i}$ denotes the fraction of the sunlight with respect to the normal direction of the facet as

$$
f_{i}=\left\{\begin{array}{cl}
0 & \text { if } \quad \boldsymbol{s}^{T} \boldsymbol{n} \leq 0 \\
\boldsymbol{s}^{T} \boldsymbol{n} & \text { elsewhere }
\end{array}\right.
$$

For all facets, the distance between the observer and the facet $r_{\mathrm{obs}}$ is assumed to be the same as the distance from the observer to the center of mass of the object, because the relative distances between the center of facets and the center of mass are small enough compared to the distance from the observer. 


\section{Solar Radiation Pressure (SRP)}

The SRP acting on a facet is also formulated using BRDF as

$$
\begin{aligned}
f_{\mathrm{SRP}} & =-\int_{0}^{\infty} \frac{F(\lambda) A f_{i}}{c}\left(s+\iint f_{r} \cos \theta_{r} \sin \theta_{r} v \mathrm{~d} \theta_{r} \mathrm{~d} \phi_{r}\right) \mathrm{d} \lambda \\
& =-\frac{S_{0}}{c r_{\mathrm{AU}}^{2}} A f_{i}\left(s+\iint f_{r} \cos \theta_{r} \sin \theta_{r} v \mathrm{~d} \theta_{r} \mathrm{~d} \phi_{r}\right)
\end{aligned}
$$

where $F(\lambda)$ is the solar flux, $c$ is the light speed, $\lambda$ is the wave length, $S_{0}$ is the solar constant, and $r_{\mathrm{AU}}$ is the distance between object and Sun in astronomical units, respectively. Under the assumption of Lambertian diffuse and mirror-like specular, the substitution of Eqs. (6), (9), and (10) into Eq. (25) yields

$$
f_{\mathrm{SRP}}=-\frac{S_{0}}{c r_{\mathrm{AU}}^{2}} A f_{i}\left\{s+\iint\left\{d \frac{\rho}{\pi}+\left[2 s F_{0} \delta\left(\sin ^{2} \theta_{i}-\sin ^{2} \theta_{r}\right) \delta\left(\phi_{i}-\phi_{r} \pm \pi\right)\right]\right\} \cos \theta_{r} \sin \theta_{r} v \mathrm{~d} \theta_{r} \mathrm{~d} \phi_{r}\right\}
$$

Since $v=\left[\sin \theta_{r} \cos \phi_{r}, \sin \theta_{r} \sin \phi_{r}, \cos \theta_{r}\right]^{T}$, the integrations for diffuse and specular terms are calculated as

$$
\begin{aligned}
& \int_{0}^{2 \pi} \int_{0}^{\frac{\pi}{2}} d \frac{\rho}{\pi} \cos \theta_{r} \sin \theta_{r} v \mathrm{~d} \theta_{r} \mathrm{~d} \phi_{r}=\frac{2}{3} d \rho \boldsymbol{n} \\
& \int_{0}^{2 \pi} \int_{0}^{\frac{\pi}{2}} 2 s F_{0} \delta\left(\sin ^{2} \theta_{i}-\sin ^{2} \theta_{r}\right) \delta\left(\phi_{i}-\phi_{r} \pm \pi\right) \cos \theta_{r} \sin \theta_{r} \boldsymbol{v} \mathrm{d} \theta_{r} \mathrm{~d} \phi_{r} \\
& =\int_{0}^{2 \pi} \int_{0}^{1} s F_{0} \delta\left(\sin ^{2} \theta_{i}-\sin ^{2} \theta_{r}\right) \delta\left(\phi_{i}-\phi_{r} \pm \pi\right) \boldsymbol{v} \mathrm{d}\left(\sin ^{2} \theta_{r}\right) \mathrm{d} \phi_{r} \\
& =s F_{0}\left[2\left(\boldsymbol{s}^{T} \boldsymbol{n}\right) \boldsymbol{n}-\boldsymbol{s}\right]
\end{aligned}
$$

where $2\left(\boldsymbol{s}^{T} \boldsymbol{n}\right) \boldsymbol{n}-\boldsymbol{s}$ is the perfect specular direction that occurs when $\sin ^{2} \theta_{i}=\sin ^{2} \theta_{r}$ and $\phi_{i}=\phi_{r} \pm \pi$. Furthermore, considering the thermal emissive term $\kappa c_{\mathrm{abs}}$, the general form of SRP is obtained using Eqs. (7), (8), and (27)-(29) as

$$
f_{\mathrm{SRP}}=-\frac{S_{0}}{c r_{\mathrm{AU}}^{2}}\left\{\left(\boldsymbol{s}^{T} \boldsymbol{n}\right)\left(c_{\mathrm{abs}}+c_{\mathrm{diff}}\right) \boldsymbol{s}+\left(\boldsymbol{s}^{T} \boldsymbol{n}\right)\left[\frac{2}{3} c_{\mathrm{diff}}+\kappa c_{\mathrm{abs}}+2 c_{\mathrm{spec}}\left(\boldsymbol{s}^{T} \boldsymbol{n}\right)\right] \boldsymbol{n}\right\} A
$$

where $\kappa$ is the thermal emissivity and the relation $c_{\mathrm{abs}}+c_{\mathrm{spec}}+c_{\mathrm{diff}}=1$ is used.

\section{Dynamics of space object}

A space object is assumed to be rigid body and have constant surface parameters in this paper. The object position is expressed with Earth-Centered Inertial (ECI) frame whose $X$ axis directs to the vernal equinox, the $Z$ axis corresponds with the rotational axis of the Earth, and the $Y$ axis lies to consist the right-handed coordinate frame. The body-fixed frame of the space object $\left\{x_{b}, y_{b}, z_{b}\right\}$ has its origin at the center of mass and the body-fixed axes correspond with the principal axes of inertia. External disturbance is assumed to be SRP forces and torques. The equations of motion of the 
space object are summarized as

$$
\begin{aligned}
\ddot{r}_{\mathrm{pos}} & =-\frac{\mu}{r_{\mathrm{pos}}^{3}} \boldsymbol{r}_{\mathrm{pos}}+F_{\mathrm{SRP}} \\
\frac{\mathrm{d}}{\mathrm{d} t}\left[\begin{array}{c}
\alpha \\
\delta \\
\psi
\end{array}\right] & =\left[\begin{array}{ccc}
\frac{\sin \psi}{\cos \delta} & \frac{\cos \psi}{\cos \delta} & 0 \\
-\cos \psi & \sin \psi & 0 \\
-\tan \delta \sin \psi & -\tan \delta \cos \psi & 1
\end{array}\right]\left[\begin{array}{c}
\omega_{x} \\
\omega_{y} \\
\omega_{z}
\end{array}\right] \\
J \dot{\omega}+\omega \times J \omega & =T_{\mathrm{SRP}}
\end{aligned}
$$

where $\boldsymbol{r}_{\mathrm{pos}}$ is the object position in ECI frame, $\mu$ is the Earth's gravitational constant, $F_{\mathrm{SRP}}$ is the sum of the SRP forces on each facet, $T_{\mathrm{SRP}}$ is the sum of the SRP torques on each facet, and $\omega=\left[\omega_{x}, \omega_{y}, \omega_{z}\right]^{T}$ is angular velocity with respect to the body-fixed frame, respectively. The attitude angles $\alpha$ and $\delta$ are the right ascension and the declination of the spin axis, and $\psi$ is the rotational angle around the spin axis. For example, the attitude angles, $\alpha, \delta$, and $\psi$, correspond with Euler angles with the $3-2-3$ sequence as $\left(\theta_{1}, \theta_{2}, \theta_{3}\right)=\left(\alpha, \frac{\pi}{2}-\delta, \psi+\frac{\pi}{2}\right)$.

\section{Approximation Method}

This section relates the formulation of light curves to the dynamics of a spinning solar sail. These formulations are expressed with common parameters, and a correction parameter that compensates the difference between the light curves and the dynamics is derived. It is noted that, although the light curve formulation is combined with the solar sail dynamics, without loss of generality, the following formulation is applicable for any spinning rigid objects.

\section{A. Generalized Sail Dynamics Model [11]}

Generalized sail dynamics model shown in [10, 11] is briefly summarized in this subsection. A space object is assumed to be spinning around the body-fixed $z_{b}$ axis. The position vector of a small facet of the object with respect to the body-fixed frame is described as

$$
\boldsymbol{r}=\left[\begin{array}{c}
r \cos \phi \\
r \sin \phi \\
z(r, \phi)
\end{array}\right]
$$


where $r$ and $\phi$ are the projected distance onto the $x_{b}-y_{b}$ plane and the phase in the $x_{b}-y_{b}$ plane, respectively. The height $z$ is assumed to be known as the function of the $r$ and $\phi$. The normal vector of the facet is written as follows.

$$
\boldsymbol{n}=\frac{\partial \boldsymbol{r}}{\partial r} \times \frac{\partial \boldsymbol{r}}{r \partial \phi}=\left[\begin{array}{c}
-\frac{\partial z}{\partial r} \cos \phi+\frac{\partial z}{r \partial \phi} \sin \phi \\
-\frac{\partial z}{\partial r} \sin \phi-\frac{\partial z}{r \partial \phi} \cos \phi \\
1
\end{array}\right]
$$

Since the SRP torque acting on the facet is written as $\tau_{\mathrm{SRP}}=\boldsymbol{r} \times \boldsymbol{f}_{\mathrm{SRP}}$, substituting Eqs. (34) and (35) into Eq. 25) yields a general form of the SPR torque on the facet. Integrating $\boldsymbol{\tau}_{\mathrm{SRP}}$ for all facets, i.e., along the radial direction and circumferential direction, provides the total SRP torque acting on the space object as

$$
\boldsymbol{T}_{\mathrm{SRP}}=\iint \boldsymbol{\tau}_{\mathrm{SRP}} r \mathrm{~d} \phi \mathrm{d} r
$$

By averaging the SRP torque with respect to a spin period, the spin averaged torque $\overline{\boldsymbol{T}}_{\mathrm{SRP}}$ is obtained as [10, 11]

$$
\overline{\boldsymbol{T}}_{\mathrm{SRP}}=\left[\begin{array}{ccc}
A & -B & 0 \\
B & A & 0 \\
0 & 0 & C
\end{array}\right]\left[\begin{array}{c}
s_{x} \\
s_{y} \\
s_{z}
\end{array}\right]
$$

where

$$
\begin{aligned}
A & =\frac{1}{2} \iint\left(2 p_{n 1}+p_{n 2}+p_{s}\right) r \frac{\partial z}{\partial \phi} \mathrm{d} \phi \mathrm{d} r \\
B & =\frac{1}{2} \iint\left(2 p_{n 1}+p_{n 2}+p_{s}\right) r^{2} \frac{\partial z}{\partial r} \mathrm{~d} \phi \mathrm{d} r+\iint p_{s} r z \mathrm{~d} \phi \mathrm{d} r \\
C & =\iint\left(p_{n 1}+p_{n 2}\right) \frac{\partial z}{\partial \phi} r \mathrm{~d} \phi \mathrm{d} r \\
p_{n 1} & =\frac{S_{0}}{c r_{\mathrm{AU}}^{2}}\left(2 c_{\mathrm{spec}}\right) \\
p_{n 2} & =\frac{S_{0}}{c r_{\mathrm{AU}}^{2}}\left(\frac{2}{3} c_{\mathrm{diff}}+\kappa c_{\mathrm{abs}}\right) \\
p_{s} & =\frac{S_{0}}{c r_{\mathrm{AU}}^{2}}\left(c_{\mathrm{abs}}+c_{\mathrm{diff}}\right)
\end{aligned}
$$

As shown in Eqs. 38-43, the parameters $A, B$, and $C$ are the functions of optical parameters and shape of the whole object. These parameters $A, B$, and $C$ are physically interpreted as torsion, warp, and radial torsion, respectively (for more detailed description, see [11]). It is noted that, if the object shape and surface parameters are time invariant for a certain distance $r_{\mathrm{AU}}$, the parameters $A, B$, and $C$ become constant. Since they include geometrical and optical 
information of the object, these three parameters thus would be also useful for describing light curves.

\section{B. Spin averaging of light curves and correction parameter}

Since the generalized sail dynamics model is derived with spin averaging, the light curve in this paper is also formulated by averaging Eq. 23] with respect to a spin period as

$$
\bar{f}_{\mathrm{obs}}=\left[2 c_{\mathrm{spec}} F_{0} \delta\left(\sin ^{2} \theta_{i}-\sin ^{2} \theta_{r}\right) \delta\left(\phi_{i}-\phi_{r} \pm \pi\right) v_{z}+\frac{c_{\mathrm{diff}}}{\pi} v_{z} s_{z}\right] A
$$

The total spin-averaged light curve of an object thus is written by

$$
\bar{F}_{\mathrm{obs}}=\iint\left(2 c_{\mathrm{spec}} F_{0} \delta\left(\sin ^{2} \theta_{i}-\sin ^{2} \theta_{r}\right) \delta\left(\phi_{i}-\phi_{r} \pm \pi\right)+\frac{c_{\mathrm{diff}}}{\pi} s_{z}\right) v_{z} r \mathrm{~d} r \mathrm{~d} \phi
$$

Since the specular reflection occurs only when $\boldsymbol{v}=2\left(\boldsymbol{s}^{T} \boldsymbol{n}\right) \boldsymbol{n}-\boldsymbol{s}$, ignoring the specular term in Eq. (45) simplifies the spin averaged light curves as

$$
\bar{F}_{\text {obs }}=\frac{s_{z} v_{z}}{\pi} \iint c_{\text {diff }} r \mathrm{~d} r \mathrm{~d} \phi
$$

Equations 38 and 40 can cancel out the specular term $p_{n 1}$, yielding

$$
\begin{aligned}
2 A-2 C & =\iint\left(p_{s}-p_{n 2}\right) \frac{\partial z}{\partial \phi} r \mathrm{~d} r \mathrm{~d} \phi \\
& =\frac{S_{0}}{c r_{\mathrm{AU}}^{2}} \iint\left[\frac{1}{3} c_{\mathrm{diff}}+(1-\kappa) c_{\mathrm{abs}}\right] \frac{\partial z}{\partial \phi} r \mathrm{~d} r \mathrm{~d} \phi
\end{aligned}
$$

Furthermore the absorption is assumed to be zero $c_{\mathrm{abs}} \approx 0$, which simplifies Eq. (48) to

$$
2 A-2 C=\frac{S_{0}}{c r_{\mathrm{AU}}^{2}} \iint \frac{1}{3} c_{\mathrm{diff}} \frac{\partial z}{\partial \phi} r \mathrm{~d} r \mathrm{~d} \phi
$$

As shown in Eqs. (46) and (49), the spin averaged light curves and the parameters in the generalized sail dynamics model, $A$ and $C$, have a similar form. The difference stems from the term $\frac{\partial z}{\partial \phi}$ that depends on the object shape only. The difference between Eqs. 46 and 49, is compensated by a correction parameter $\xi$. The parameter $\xi$ corrects the difference as

$$
\begin{aligned}
2 A-2 C & =\frac{S_{0}}{c r_{\mathrm{AU}}^{2}} \iint \frac{1}{3} c_{\mathrm{diff}} \frac{\partial z}{\partial \phi} r \mathrm{~d} r \mathrm{~d} \phi \\
& =\frac{S_{0}}{c r_{\mathrm{AU}}^{2}} \frac{\pi}{s_{z} v_{z}} \bar{F}_{\mathrm{obs}} \xi
\end{aligned}
$$


In Eq. [51], the correction parameter is introduced that corresponds with the difference due to $\frac{\partial z}{r \partial \phi}$. It is noted that this correction parameter does not depend on the surface parameter of the object, but the shape of the object. Thus the correction parameter needs to be calculated only once. Given that the distance $r_{\mathrm{AU}}$ is known, the parameters $A$ and $C$ become constant, and their calculation is also needed once. The light curve term $\bar{F}_{\text {obs }}$ is consequently approximated as follows.

$$
\bar{F}_{\text {obs }}=(2 A-2 C) \frac{c r_{\mathrm{AU}}^{2}}{S_{0} \xi} \frac{s_{z} v_{z}}{\pi}
$$

It is also noted that although this approximation is obtained ignoring specular reflections in light curves, this does not mean the specular term in SRP is assumed to be zero. That is, the specular reflection in SRP is considered in the dynamics, and both parameters $A$ and $C$ are used in the formulation in Eq. 48.

\section{Observability}

The parameters $A, B$, and $C$ can be obtained by calculating spin averaged SRP torques in Eq. (37), when a space object model is available. These parameters, however, may vary due to contamination and/or shape deformation, and it is practically useful that they are included in state vector and estimated simultaneously. Assuming the approximation in Eq. (52) is true, the static observability of $A$ and $C$ is examined with respect to light curve observations. The sensitivity of the parameters $A$ and $C$ is calculated using Eq. (52) as

$$
\begin{gathered}
\frac{\partial \bar{F}_{\mathrm{obs}}}{\partial A}=2 \frac{c r_{\mathrm{AU}}^{2}}{S_{0} \xi} \frac{s_{z} v_{z}}{\pi} \\
\frac{\partial \bar{F}_{\mathrm{obs}}}{\partial C}=-2 \frac{c r_{\mathrm{AU}}^{2}}{S_{0} \xi} \frac{s_{z} v_{z}}{\pi}
\end{gathered}
$$

It is obvious that the sensitivity due to the parameter error of $A$ is the same as that due to the parameter error of $C$ except for their signs. Thus increasing $A$ is the same as decreasing $C$ and vice versa, which indicates that the parameters $A$ and $C$ are not simultaneously observable. This result is interestingly similar to that shown in [12] which describes that the surface parameters $c_{s}$ and $c_{d}$ are not jointly observable. This unobservability may be avoided by considering polarized light curve observations as shown in [13]. For an almost-flat object, the parameter $C$ has much smaller value than the parameter $A$, and the parameter $A$ is observable ignoring the effect of the parameter $C$.

\section{Applicability of other BRDFs}

The proposed approximation method is based on the simple BRDF that assumes the Lambertian diffuse and perfect specular. Although applying other BRDFs such as Cook-Torrance model and Ashikhmin-Shirley model may provide more detailed light curves and SRP, these BRDFs make the problem more complicated. Thus the applicability of these 
BRDFs is briefly described here, and further analysis should be conducted in future works.

Applying other BRDFs requires to redefine the generalized sail dynamics, and its difficulty stems from that the integration in Eq. 25] cannot be obtained analytically for Cook-Torrance model and Ashikhmin-Shirley model. Especially the formulations of specular reflection complicates the integration as shown in Eqs. (12) and (20). Nevertheless, numerical integrations show that the specular component of the SRP vector does not deviate much from the perfect specular direction. To this end, an offset angle is defined as the angle between the perfect specular direction and the specular reflection direction that is numerically obtained. For example, the offset angle for Cook-Torrance model is defined as

$$
\theta_{\mathrm{CT}}=\arccos \left(\boldsymbol{v}_{\mathrm{ref}}^{T} \overline{\boldsymbol{v}}_{\mathrm{CT}}\right)
$$

where

$$
\begin{aligned}
\boldsymbol{v}_{\mathrm{ref}} & =2\left(\boldsymbol{n}^{T} \boldsymbol{s}\right) \boldsymbol{n}-\boldsymbol{s} \\
\overline{\boldsymbol{v}}_{\mathrm{CT}} & =\frac{\boldsymbol{v}_{\mathrm{CT}}}{\left\|\boldsymbol{v}_{\mathrm{CT}}\right\|} \\
\boldsymbol{v}_{\mathrm{CT}} & =\iint \frac{D G F}{4\left(\boldsymbol{n}^{T} \boldsymbol{s}\right)\left(\boldsymbol{n}^{T} \boldsymbol{v}\right)} \cos \theta_{r} \sin \theta_{r} \boldsymbol{v} \mathrm{d} \theta_{r} \mathrm{~d} \phi_{r}
\end{aligned}
$$

The offset angle for Ashikhmin-Shirley model $\theta_{\text {AS }}$ is similarly defined using Eq. 201. Figures 2 and 3 show the offset angles of Cook-Torrance model and Ashikhmin-Shirley model, respectively, and they indicate the deviation of the specular directions from the perfect specular direction is less than $15 \mathrm{deg}$, except for large value of $m$ and small values of $n_{u}$ and $n_{v}$. These small offset angles are due to cancelling out in the integration of the specular components around the perfect specular direction $v_{\text {ref. }}$. The applicability of other BRDFs to the proposed method requires to numerically compensate for the offset when $m$ is large or $n_{u}$ and $n_{v}$ are small, which would provides semi-analytical direction of the SRP vector for Cook-Torrance model and Ashikhmin-Shirley model. Then generalized sail dynamics should be redefined using Cook-Torrance model or Ashikhmin-Shirley model.

\section{Numerical Examples}

This section shows numerical examples for the flat cone model, the box-wing model, and the box-wing model with large radial torsion. These simulations are conducted with the initial orbital and attitude condition described in Table 1 The light curve observations are assumed to be obtained in every one second. The orbital position and attitude of the object is assumed to be known to verify the approximation accuracy in this paper. Although a flat plate is the simplest model often used [5, 8], such complete flat object cannot not be expressed with the generalized sail dynamics model, because the parameters $A, B$, and $C$ become zero as shown in Eqs. (38)-40]. 


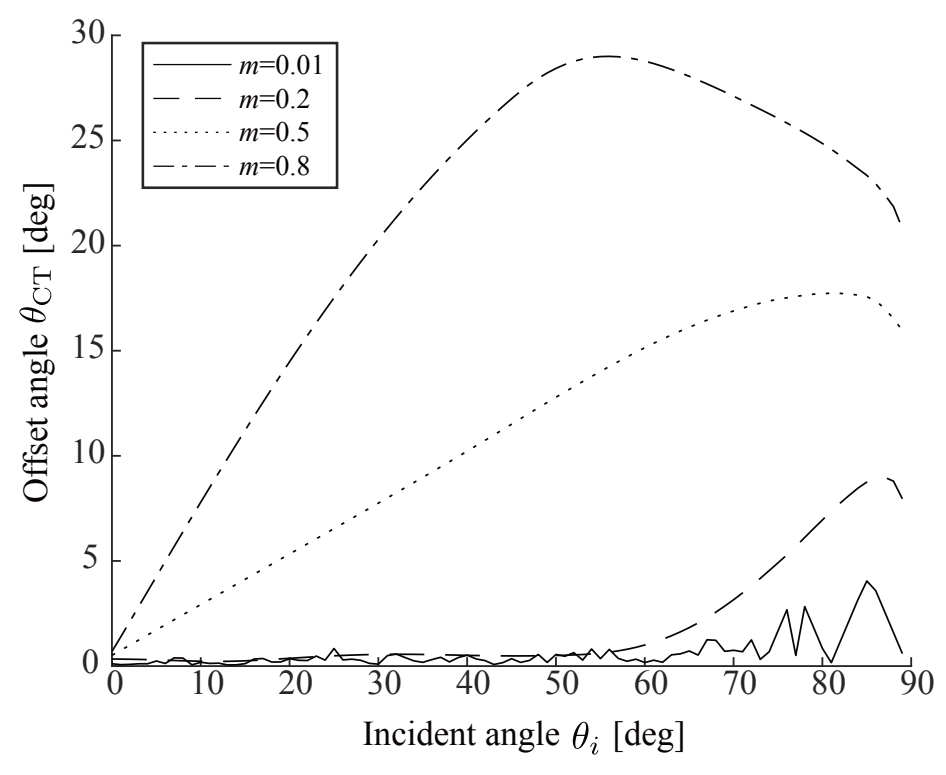

Fig. 2 Offset angle of Cook-Torrance model

Table 1 Initial condition

\begin{tabular}{lc}
\hline \hline Parameter & Value \\
\hline Position (inertial frame), $\boldsymbol{r}$ & $(19199.346,37540.319,24.873) \mathrm{km}$ \\
Velocity (inertial frame), $\boldsymbol{r}$ & $(-2.737,1.401,-0.002) \mathrm{km} / \mathrm{s}$ \\
Attitude angle, $(\alpha, \delta, \psi)$ & $(-40.0,0.0,0.0) \mathrm{deg}$ \\
Angular velocity, $\left(\omega_{x}, \omega_{y}, \omega_{z}\right)$ & $(0.0,0.2,-1.0) \mathrm{deg} / \mathrm{s}$ \\
\hline \hline
\end{tabular}

\section{A. Flat Cone Model}

A flat cone model as shown in Fig. 4 is considered. This model is split into 20 facets along radius direction and 60 facets along circumferential direction for the high-fidelity calculation of SRP and light curves. The model size, surface parameters, and corresponding $A, B$, and $C$ parameters are summarized in Table 2

Figure 5 shows the time history of true light curve and approximated one, and the approximation successfully corresponds with the true value. After $4000 \mathrm{~s}$, the sudden change of the light curve, called glint, appears. The glint effect is not approximated in the proposed method, because the glint causes due to the specular reflection and the approximation ignores it in the formulation. For a simple model, the magnitude of the light curve is accurately approximated with a fewer number of parameters as described in Eq. 52 .

\section{B. Box-wing model}

Next, a box-wing model as more general shape of spacecraft is considered and the model is illustrated in Fig.6. The box wing model consists of the satellite body (box) and the two solar array panels (wings). As noted above, since complete flat object is not described with generalized sail dynamics model, the solar array panels of the box wing 


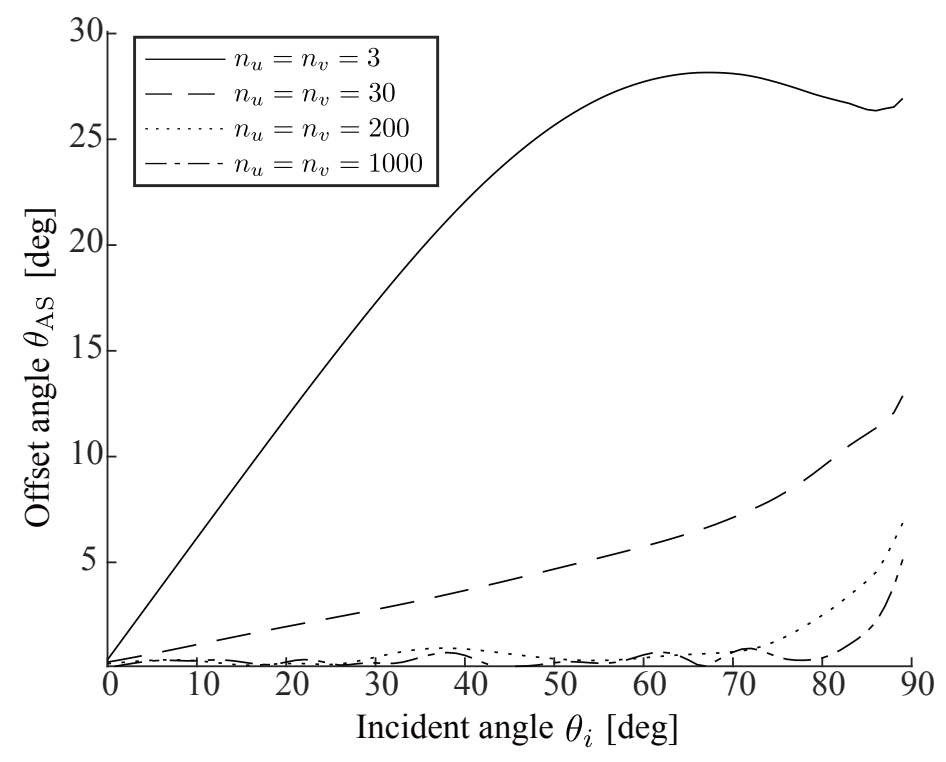

Fig. 3 Offset angle of Ashikhmin-Shirley model

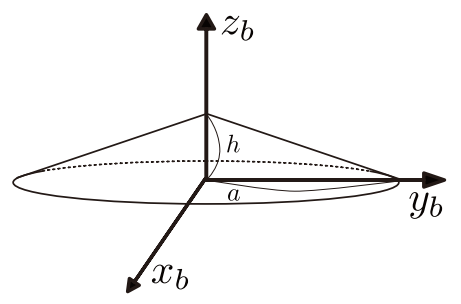

Fig. 4 Flat cone model

model are rotated at 10 deg along $y_{b}$-axis in this numerical simulation. Each surface of both box and wings is split into 100 small facets for the high-fidelity calculation of SRP and light curves. The geometric model parameters and the corresponding $A, B$, and $C$ parameters are described in Table 3

Figure 7 shows the time history of the true light curve and approximated one. The order of the light curve and its low frequent changes are successfully approximated. The high frequent part and glint effect of the light curve, however, are not realized with the proposed approximation. The true light curve history is more complicated than that of the flat cone model, because the box-wing model has three components and two different surface parameters. On the other hand, the approximation is the function of the third component of the sun direction and observer one as shown in Eq. 52. Thus, the approximation does not completely realize the complicated true light curves. Although the proposed approximation ignores the absorption parameter, the parameters of the box-wing model have absorption parameter as shown in Table 3 Nevertheless, the approximation result in Fig. 7 has reasonable consistency with respect to true one. As mentioned in Introduction, detailed object modeling requires many shape and optical parameters, whereas the proposed method uses only two parameters $A$ and $C$. For attitude estimation using light curves, the trade-off study between the accuracy of the 
Table 2 Flat cone model parameters

\begin{tabular}{lc}
\hline \hline Parameter & Value \\
\hline Height, $h$ & $0.2 \mathrm{~m}$ \\
Radius, $a$, & $2.0 \mathrm{~m}$ \\
Surface parameters, $\left(c_{\text {abs }}, c_{\text {diff }}, c_{\text {spec }}\right)$ & $(0.0,0.16,0.84)$ \\
Parameters, $A, B, C$ & $\left(1.854 \times 10^{-6}, 1.854 \times 10^{-6}, 1.455 \times 10^{-21}\right)$ \\
Moment of inertia $\left(J_{x}, J_{y}, J_{z}\right)$ & $(100,100,150) \mathrm{kgm}^{2}$ \\
\hline \hline
\end{tabular}

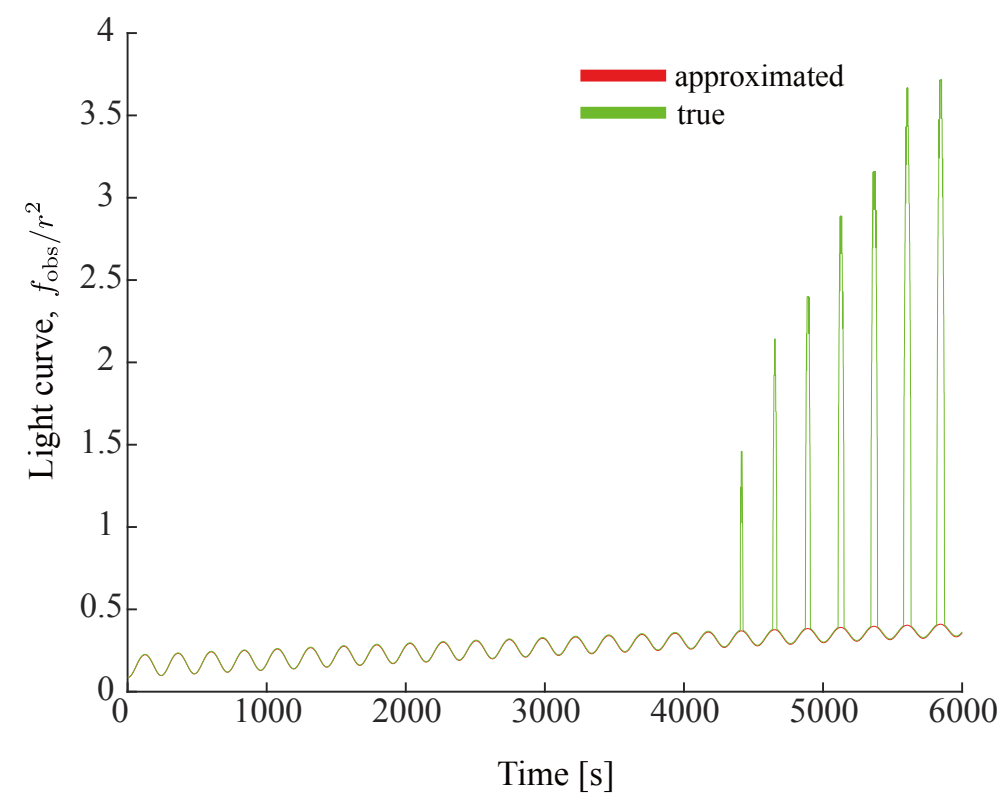

Fig. 5 Approximated and true light curves for flat cone model

light curves and the number of parameters should be qualitatively conducted.

\section{Box-wing model with large radial torsion}

The previous examples have larger value of $A$ than that of $C$, and the contribution of the parameter $C$ is difficult to understand by their approximation results. The difference between $A$ and $C$ mainly stems from the shape of the object. As mentioned above, the parameter $C$ physically describes the radial torsion of the object. Thus a box-wing model with large radial torsion is considered. That is, the wings are modeled as $\left(\theta_{\text {wing, }+y_{b}}, \theta_{\text {wing, }-y_{b}}\right)=(30,-30)$ so that the radial torsion becomes larger than the previous box-wing model, resulting in large value of $C$. The other geometrical and optical parameters are the same as written in Table. 3 Setting the wing rotation to $\left(\theta_{\text {wing, }+y_{b}}, \theta_{\text {wing, }-y_{b}}\right)=(30,-30)$ deg provides the parameters $(A, B, C)=\left(5.565 \times 10^{-7}, 5.565 \times 10^{-7}, 7.280 \times 10^{-7}\right)$, and all parameters have the similar order as expected.

Figure 8 demonstrates the approximated light curves and true one. Although glint and high-frequent changes are not 




Fig. 6 Box-wing model

Table 3 Box-wing model parameters

\begin{tabular}{lc}
\hline \hline Parameter & Value \\
\hline Box length, $\left(a_{\text {box }}, b_{\text {box }}, c_{\text {box }}\right)$ & $(0.5,0.5,0.8) \mathrm{m}$ \\
Wing length, $\left(a_{\text {wing }}, b_{\text {wing }}, c_{\text {wing }}\right)$, & $(0.5,1.0,0.05) \mathrm{m}$ \\
Wing ration angle, $\left(\theta_{\text {wing, }+y_{b}}, \theta_{\text {wing, }-y_{b}}\right)$ & $(10,10) \mathrm{deg}$ \\
Surface parameters, $\left(c_{\text {abs }}, c_{\text {diff }}, c_{\text {spec }}\right)$ & \\
Box: & $(0.4,0.2,0.4)$ \\
Wing: & $(0.1,0.6,0.3)$ \\
Parameters, $A, B, C$ & $\left(5.565 \times 10^{-7}, 5.565 \times 10^{-7}, 1.818 \times 10^{-23}\right)$ \\
Moment of inertia $\left(J_{x}, J_{y}, J_{z}\right)$ & $(80,80,150) \mathrm{kgm}^{2}$ \\
\hline \hline
\end{tabular}

approximated in the same reason to the previous results, the proposed formulation successfully approximates the light curves. This result thus verifies the effectiveness of Eq. 52 when both parameters $A$ and $C$ have the same order and equally contribute to the approximation.

\section{Conclusions}

An light curve approximation method for the state estimate of a space object is addressed in this paper. Motivated by a generalized spinning sail model that uses only three parameters, this study combines them with light curve formulation, reducing the number of state variables to express light curves. Numerical examples show the proposed formulation accurately approximate the light curve of a simple flat cone model. Although, for more complicated object such as a box wing model, high frequent changes of the light curve are not approximated, the proposed method shows the reasonable correspondence with low frequent changes of the light curve using a fewer number of parameters. 


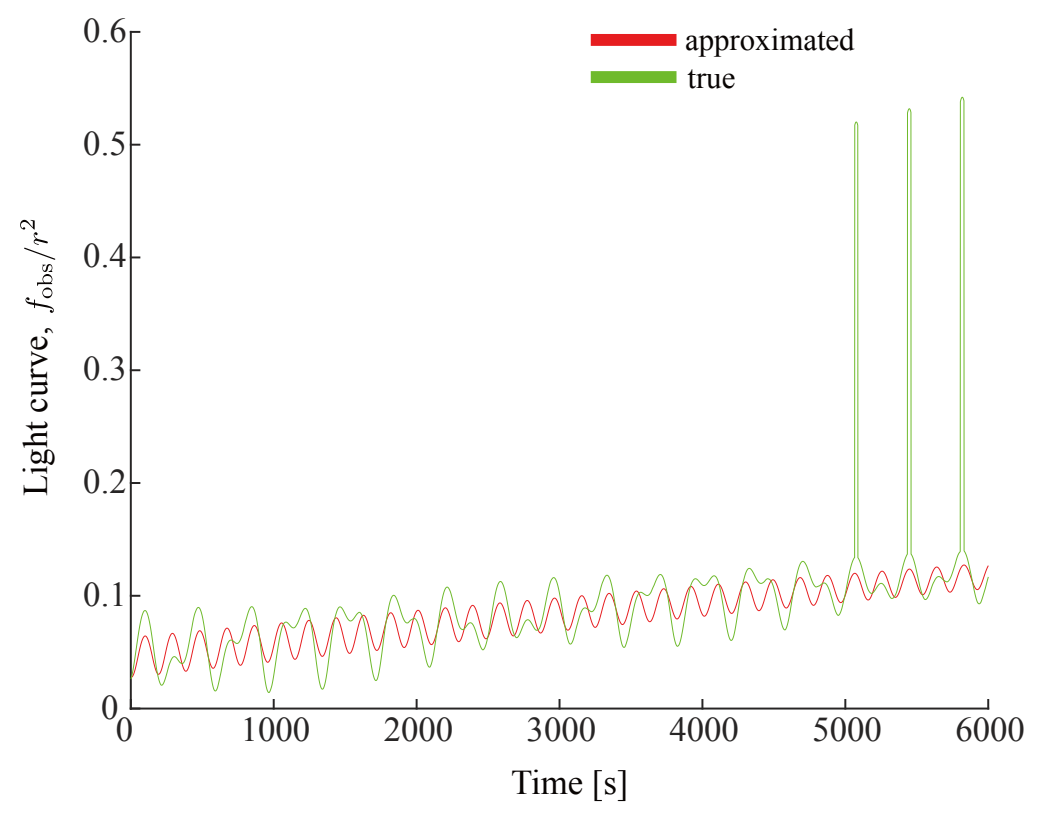

Fig. 7 Approximated and true light curves for box-wing model

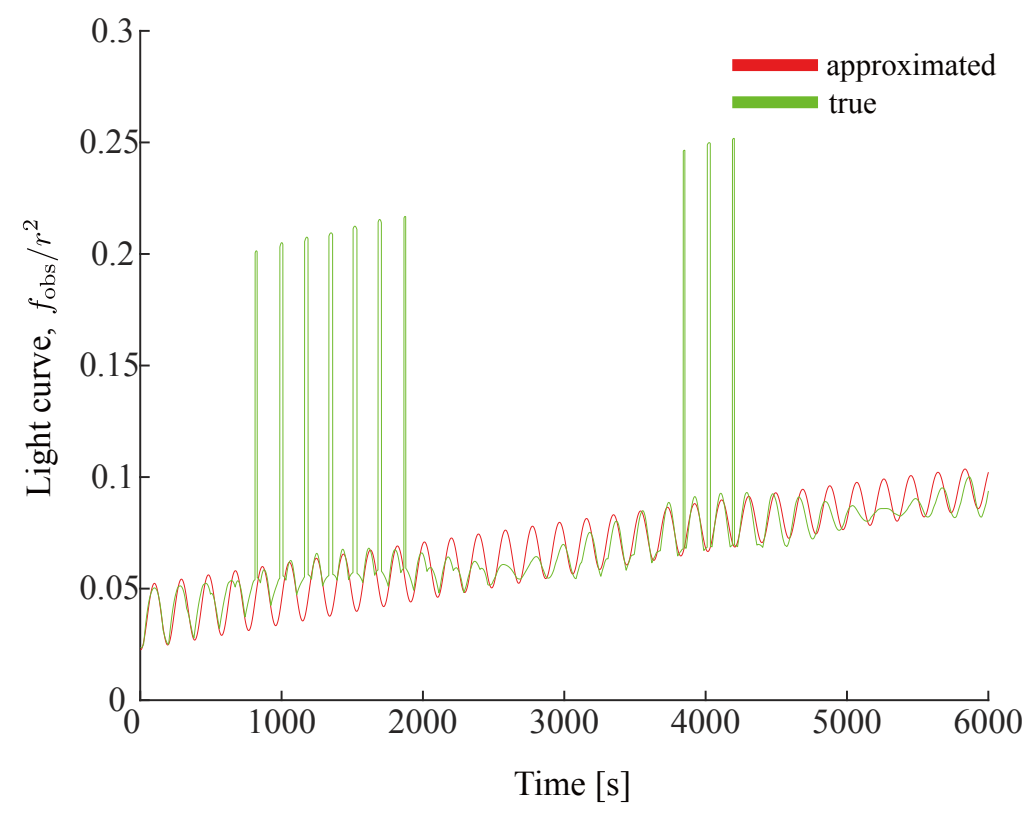

Fig. 8 Approximated and true light curves for box-wing model with large radial torsion

\section{Acknowledgement}

This research was partially supported by the Kajima Foundation's Assistance for Exchange of Researcher. 


\section{References}

[1] Kennewell, J. A., and Vo, B.-N., “An Overview of Space Situational Awareness,” 2013 16th International Conference on Information Fusion (FUSION), IEEE, Piscataway, NJ, 2013, pp. 1029-1036.

[2] Bobrinsky, N., and Del Monte, L., "The space situational awareness program of the European Space Agency," Cosmic Research, Vol. 48, No. 5, 2010, pp. 392-398. doi:10.1134/S0010952510050035.

[3] McInnes, C. R., Solar Sailing: Technology, Dynamics and Mission Applications, Springer-Praxis, Chichester, England, U.K., 1999, pp. 32-55.

[4] Cook, R. L., and Torrance, K. E., "A Reflectance model for Computer Graphics," ACM Transactions on Graphics (TOG), Vol. 1, 1982, pp. 7-24.

[5] Kelecy, T., Hussein, I. I., Miller, K., and Coughlin, J., "Probabilistic Analysis of Light Curves,” The Journal of the Astronautical Sciences, Vol. 66, No. 2, 2019, pp. 142-161. doi:10.1007/s40295-018-0130-3.

[6] Ashikhmin, M., and Shirley, P., “An Anisotropic Phong BRDF Model,” Journal of graphics tools, Vol. 5, No. 2, 2000 , pp. 25-32. doi:10.1080/10867651.2000.10487522.

[7] Wetterer, C. J., Linares, R., Crassidis, J. L., Kelecy, T. M., Ziebart, M. K., Jah, M. K., and Cefola, P. J., "Refining Space Object Radiation Pressure Modeling with Bidirectional Reflectance Distribution Functions," Journal of Guidance, Control, and Dynamics, Vol. 37, No. 1, 2014, pp. 185-196. doi:10.2514/1.60577.

[8] Mallik, V., and Jah, M. K., "Reconciling space object observed and solar pressure albedo-areas via astrometric and photometric data fusion," Advances in Space Research, Vol. 63, No. 1, 2019, pp. 404-416. doi:10.1016/j.asr.2018.08.005.

[9] Funase, R., Shirasawa, Y., Mimasu, Y., Mori, O., Tsuda, Y., Saiki, T., and Kawaguchi, J., “On-orbit verification of fuel-free attitude control system for spinning solar sail utilizing solar radiation pressure,” Advances in Space Research, Vol. 48, No. 11, 2011, pp. 1740-1746. doi:10.1016/j.asr.2011.02.022.

[10] Tsuda, Y., Saiki, T., Funase, R., and Mimasu, Y., “Generalized Attitude Model for Spinning Solar Sail Spacecraft,” Journal of Guidance, Control, and Dynamics, Vol. 36, No. 4, 2013, pp. 967-974. doi:10.2514/1.59516.

[11] Ono, G., Tsuda, Y., Akatsuka, K., Saiki, T., Mimasu, Y., Ogawa, N., and Terui, F., "Generalized Attitude Model for Momentum-Biased Solar Sail Spacecraft," Journal of Guidance, Control, and Dynamics, Vol. 39, No. 7, 2016 , pp. 1491-1500. doi:10.2514/1.G001750.

[12] Hinks, J. C., Linares, R., and Crassidis, J. L., "Attitude Observability from Light Curve Measurements,” AIAA Guidance, Navigation, and Control (GNC) Conference, AIAA Paper 2013-5005, 2013. doi:10.2514/6.2013-5005.

[13] Dianetti, A., and Crassidis, J., "Space Object Material Determination from Polarized Light Curves," AIAA Scitech 2019 Forum, AIAA, 2019. doi:10.2514/6.2019-0377. 